\title{
Los Programas de Promoción de Empleo: UN BALANCE A LA GESTIÓN DE TRES LEGISLATURAS DEL Gobierno ANDALuZ
}

\author{
Soledad Barrios Martinez \\ Juan Delgado Alaminos \\ Universidad de Granada
}

En este trabajo se presentan las diferentes actuaciones del gobierno autonómico andaluz en materia de promoción de empleo. En una primera parte, se muestran los distintos rasgos que han definido la politica de intervención directa del ejecutivo andaluz en el mercado de trabajo, en el que se integran tanto medidas orientadas a la demanda de trabajo como a la oferta. Posteriormente, se presenta un balance final de la evolución del mercado de trabajo andaluz del que se deriva una serie de consideraciones sobre la política de promoción de empleo en Andalucía duranté casi tres lustros de política autonómica.

In this work are presented the different performances of the self-governing Andalusian in matter of employment promotion. In a first part, are shown the different features that they have defined the direct intervention policy of the Andalusian executive in the job market, in the one which are integrated so much measures guided to the work demand as to the offer. Thereinafter, it is presented a final balance of the evolution of the Andalusian job market of the that is derived a series of considerations on the policy of employment promotion in Andalusia during almost fifteen years of self-governing.

Palabras clave: política de empleo, mercado de trabajo, desempleo, formación.

Key words: political of employment, labour market, unemployment, training.

\section{INTRODUCCIÓN}

Cuando se aprobó el Estatuto de Autonomía de Andalucía, al final de la década de los setenta, el desempleo era uno de los principales problemas (si no el principal) de la economía andaluza. Ante tal situación, el Estatuto de Autonomía con- 
sideraba como objetivo básico (art.12.3) "la consecución del pleno empleo en todos los sectores de la_producción y la especial garantía de puestos de trabajo. para las jóvenes generaciones de andaluces". Estas aspiraciones, que se recogen en lo que se puede denominar la Carta Magna de la Comunidad Autónoma de Andalucía, hoy día, siguen siendo un importante reto para la región.

Según el marco competencial establecido tanto en la Constitución como en el Estatuto de Autonomía, la regulación del mercado laboral viene fijada por la politica de ámbito nacional, por lo que no se puede incidir en ese ámbito con políticas autonómicas. Esto no supone que el ejecutivo andaluz permanezca impasible ante los problemas del mercado laboral, ya que al gobierno autonómico se le atribuyen competencias ejecutivas ${ }^{1}$. En este sentido, éste puede desarrollar una política de empleo activa ${ }^{2}$ que puede aplicar de forma paralela con las otras administraciones. Con este tipo de política, que tiende a identificarse en palabras de Rehn (1989, p.48) "con el conjunto de programas que fomentan la creación de puestos de trabajo allí donde la demanda de trabajo es insuficiente para que haya pleno empleo (programas orientados hacia la demanda), o fomentan el reajuste de la mano de obra a las diferencias intersectoriales o interregionales y a las variaciones de la estructura de la demanda (programas orientados hacia la oferta)", el gobierno autonómico tiene un papel importante en la creación de empleo.

1. En materia laboral, el Estatuto de Autonomía atribuye expresamente competencias ejecutivas al gobierno autonómico y así vienen establecidas en el art. 17.2.: "Corresponde a la Comunidad Autónoma de Andalucía la ejecución de la legislación del Estado en materia laboral, con las facultades y servicios propios de la Administración respecto de las relaciones laborales, sin perjuicio de la alta inspección dẹl Estado y de lo establecido en el art. 149, 1, 2 de la Constitución".

2. Según la OCDE (1996), las políticas de empleo activas son la formación ocupacional, las medidas destinadas a los jóvenes; las subvenciones a la creación directa de empleo y a la contratación (quẹe pueden adoptar la forma de subvenciones directas o subvenciones implícitas derivádas, por ejemplo, de cambios legales) y las medidas en favor de los incapacitados. Estas políticas pueden incidir a traves: de diferentes medidas en el mercado laboral, tanto por el lado de la demanda como de la oferta. 
los Programas de Promoción de Empleo: Un balance a la Gestión de tres legislaturas del

\section{Cuadro $n^{\circ} 1$}

Esquema de intervención del gobierno autonómico de las políticas de fomento de empleo

\begin{tabular}{|c|c|c|c|}
\hline \multirow{9}{*}{$\begin{array}{c}\text { MEDIDAS } \\
\text { ORIENTADAS } \\
\text { A LA } \\
\text { DEMANDA DE } \\
\text { TRABAJO }\end{array}$} & \multicolumn{2}{|c|}{$\begin{array}{l}\text { 1. MEDIDAS EN FAVOR DE CONTRATACIÓN DE GRUPOS DES- } \\
\text { FAVORECIDOS. }\end{array}$} & \multirow{6}{*}{$\begin{array}{c}\text { Programa } \\
\text { de } \\
\text { Fomento } \\
\text { de } \\
\text { Empleo }\end{array}$} \\
\hline & \multirow{4}{*}{$\begin{array}{l}\text { Autofinanciadas } \\
(* *)\end{array}$} & $\begin{array}{l}\text { Programa de inserción socio laboral de } \\
\text { Jóvenes (prog. Andalucía Joven) }\end{array}$ & \\
\hline & & Parados de larga duración. & \\
\hline & & Inserción profesional. $(\bullet)$ & \\
\hline & & Subv.progr. que mantengan empleo. & \\
\hline & $\begin{array}{l}\text { En colaboración } \\
\left({ }^{*}\right)\end{array}$ & $\begin{array}{l}\text { Integración laboral de trabajadores minus- } \\
\text { válidos } \\
\text { Plan de Empleo Rural }\end{array}$ & \\
\hline & \multicolumn{2}{|c|}{$\begin{array}{l}\text { 2. MEDIDAS EN FAVOR DE INICIATIVAS EMPRESARIALES Y } \\
\text { CREACIÓN DE EMPLEO }\end{array}$} & \multirow{3}{*}{$\begin{array}{l}\text { Programa de } \\
\text { Economia Social }\end{array}$} \\
\hline & En colaboración & Promoción empleo autónomo & \\
\hline & Autofinanciadas & Fomento economía social & \\
\hline $\begin{array}{c}\text { MEDIDAS } \\
\text { ORIENTADAS } \\
\text { A LA OFERTA } \\
\text { DE TRABAJO }\end{array}$ & \multicolumn{2}{|c|}{ Formación de la mano de obra.(*) } & $\begin{array}{l}\text { Programa de } \\
\text { Formación } \\
\text { Profesional } \\
\text { Ocupacional. }\end{array}$ \\
\hline
\end{tabular}

(०) Este tipo de medidas estaban integradas hasta 1990 en el Programa presupuestario de Fomento de Empleo.

(*) Medidas autofinanciadas: son formuladas "desde y para" la región andaluza. Medidas en colaboración: son promovidas por el gobierno autonómico pero vinculadas a las medidas de empleo estatales y son financiadas via transferencias del Estado y. en otros casos, a través de convenios de colaboración con otros entes públicos.

Fuente: Elaboración propia.

Por consiguiente, el gobierno andaluz, dadas sus limitaciones competenciales, no tiene opción en el campo de la regulación del mercado laboral, pero sí tiene mucho que decir en otros campos tales como en materia de formación y en otro conjunto de medidas consistentes básicamente en subvencionar la contratación para favorecer la colocación de determinados colectivos laborales. Además, al eje- 
cutivo andaluz, en virtud de su papel regulador, le compete la responsabilidad de mantener un clima que sea favorable al funcionamiento eficiente del mercado de trabajo, aunque no tenga potestad para acometer una política salarial, por lo que deberá adoptar un papel activo en la tarea de fomentar el diálogo y la medición entre los interlocutores sociales.

Ante esto, la principal cuestión que se plantea con respecto a estas medidas se centra en la delimitación de sus efectos. En este sentido, en los siguientes apartados se describe, por un lado, los perfiles y la evolución presupuestaria de las principales medidas llevadas a cabo por el gobierno autonómico hasta mediados de la década de los años noventa,en concreto hasta 1994, periodo en el que han concurrido tres legislaturas de administración autonómica y que resulta suficientemente ilustrativo de la evolución de dicha política y permite apreciar los cambios de orientación a los que se ve sometida la política de fomento de empleo. Estas medidas se sintetizan en los programas de promoción de empleo en los que han concurrido distintos niveles administrativos para su financiación, y que responden al esquema que se presenta en el cuadro $n^{\circ} 1$. Por otro lado, paralelamente se hace un balance de la situación del mercado de trabajo, tratando de ofrecer una serie de consideraciones de interés.

\section{LOS GRANDES RASGOS QUE HAN CARACTERIZADO LA POLÍTICA DE PROMOCIÓN DE EMPLEO}

En el marco en el que se desarrolla la acción gubernamental del ejecutivo andaluz se producen una serie de actuaciones que durante el periodo inicial de administración autonómica (1982-84) se centran básicamente en el despliegue escalonado de su estructura orgánica, coincidiendo con el traspaso de competencias que se inicia en $1983^{3}$. Es a partir de 1985 cuando se empieza a desarrollar una política de promoción de empleo, aún cuando antes, desde la constitución del gobierno autonómico en agosto de 1982, se promueven un conjunto de actuaciones económicas con el propósito de crear empleó.

3. En el espacio de dos años, desde el 1 de enero de 1983 al 31 de diciembre de 1984 Andalucía había asumido en materia de Trabajo y Seguridad Social el máximo nivel de competencias de las Comunidades Autónomas con autonomia plena. Se habian transferido las competencias sobre relaciones de trabajo, seguridad e higiene, mediación, conciliación y arbitraje laboral (regulación de empleo, fondo nacional de protección al trabajo).

4. Estas primeras actuaciones, de acuerdo con las intenciones del Plan Económico para Andalucia 1984-86, se basaron en la puesta en marcha de un cierto volumen de inversiones püblicas, así como en la participación directa en la promoción y reconversión de empresas andaluzás a travês del organisisno del Instituto de Promoción Industrial para Andalucía y la empresa Sociedad para la Promocíniny Reconversión de Andalucía, con el objetivo de la creación de empleo. 
La política de promoción de empleo se ha dirigido hacia diversos campos de actuación (ver cuadro $\mathrm{n}^{\circ} 1$ ). Unas medidas se han orientado a la demanda de trabajo. Dichas acciones se han instrumentado principalmente a través de los programas presupuestarios de fomento de empleo y de fomento de la economía social. Otras actuaciones se han dirigido a mejorar la oferta de trabajo, instrumentadas en los programas de formación profesional ocupacional. Estas medidas, que han contado con un presupuesto creciente que ha pasado de representar en 1985 un $0,4 \%$ del gasto total de la Comunidad, a un $2,4 \%$ en 1997 , se convierten, a partir de entonces, en el instrumento directo del ejecutivo autonómico en la lucha contra el desempleo y en la corrección de los desequilibrios del mercado de trabajo.

Cuadro $n^{\circ} 2$

Evolución presupuestaria de los programas de fomento de empleo

(en miles de pesetas)

\begin{tabular}{lccccc}
\hline Año & $\begin{array}{l}\text { Crédito } \\
\text { Inicial }\end{array}$ & $\begin{array}{c}\text { Crédito } \\
\text { Definitivo (2) }\end{array}$ & $\begin{array}{c}\text { Obligaciones } \\
\text { Reconocidas (3) }\end{array}$ & $\begin{array}{c}\text { Ejecución } \\
\text { \%(3)/(2) }\end{array}$ & $\begin{array}{c}\text { \% del Crédito Inicial } \\
\text { sobre Presup. C.A. }\end{array}$ \\
\hline $\mathbf{1 9 8 4}$ & $\mathbf{1 . 3 9 8 . 7 2 4}$ & 1.758 .729 & 700.206 & 39.8 & 0,4 \\
$\mathbf{1 9 8 5}$ & $\mathbf{1 . 3 9 8 . 7 0 0}$ & 2.649 .168 & 1.354 .412 & 51,1 & 0,3 \\
$\mathbf{1 9 8 6}$ & 2.561 .656 & 6.123 .513 & 4.321 .354 & 70.6 & 0,5 \\
$\mathbf{1 9 8 7}$ & 5.419 .685 & 7.859 .233 & 6.560 .890 & 83,5 & 0.8 \\
$\mathbf{1 9 8 8}$ & $\mathbf{1 1 . 4 5 8 . 2 6 9}$ & 13.741 .535 & 11.503 .154 & 83,7 & 1.4 \\
$\mathbf{1 9 8 9}$ & $\mathbf{1 4 . 8 1 4 . 3 0 9}$ & 16.336 .753 & 13.808 .350 & 84.5 & 1,4 \\
$\mathbf{1 9 9 0}$ & $\mathbf{1 9 . 4 8 0 . 7 9 6}$ & $\mathbf{2 3 . 6 9 7 . 5 5 0}$ & 19.669 .975 & 83.0 & 1.6 \\
$\mathbf{1 9 9 1}$ & 27.535 .568 & $\mathbf{2 7 . 4 7 7 . 3 4 2}$ & 23.672 .224 & 86,2 & 2,0 \\
$\mathbf{1 9 9 2}$ & 30.749 .288 & 31.223 .216 & 21.537 .436 & 69.0 & 2,0 \\
$\mathbf{1 9 9 3}$ & 30.748 .671 & 42.428 .643 & 30.063 .805 & 70,9 & 1,9 \\
$\mathbf{1 9 9 4}$ & 43.630 .730 & 61.488 .482 & 41.675 .216 & 67.8 & 2,4 \\
\hline
\end{tabular}

Fuente: Presupuestos de la Comunidad Autónoma, Cuentas Cienerales de la C.A. y elaboración propia5

5. Los créditos definitivos son el resultado de incorporar al presupuesto inicial las modificaciones aprobadas a lo largo del ejercicio económico por los órganos de gobierno de la Comunidad Autónoma. Las obligaciones reconocidas son los créditos comprometidos por la Comunidad Autónoma como consecuencia del nacimiento de un derecho de un acreedor hacia ella. Los datos obtenidos sobre los créditos definitivos y obligaciones reconocidas durante el periodo 1985-1990, años durante los cuales no existía un desglose presupuestario por programas en las Cuentas generales, se han obtenido realizando un estudio comparativo de lo presupuestado y de lo realmente ejecutado. Para ello, se ha tomado como referencia cada capítulo y artículo, y se ha buscado su realización en la Cuenta General, excepto las partidas de gastos de personal y gastos corrientes que se ha considerado un grado de ejecución de un $100 \%$. 
El grado de ejecución presupuestaria de estos programas ha sido diverso. Tal como queda reflejado en el cuadro $\mathrm{n}^{\circ} 2$, en el que se muestra la evolución presu: puestaria de los programas de empleo hasta mediados de los años noventa, el cumplimiento de los presupuestos ha sido muy irregular. En general, la política de promoción de empleo no se ha caracterizado por un alto grado de cumplimiento, ya que apenas llega al $80 \%$ en la mayoría de los años. Destaca, por su baja ejecución, el periodo 92-94, con independencia de los años iniciales. No obstante, las modificaciones más importantes de los créditos iniciales en favor de los créditos o presupuestos definitivos se producen en 1993 y 1994 . La causa de tal incremento es la delicada situación económica por la que atravesaba Andalucía y que obligó al ejecutivo andaluz, casi con carácter extraordinario, a apoyar las acciones que generasen o mantuviesen empleo. Estas iniciativas estaban respaldas por un Acuerdo para el desarrollo económico y social de Andalucía firmado por la Junta de Andalucía con los agentes económicos de la región en mayo de 1993.

A continuación, se describen cada una de las medidas que han formado parte de la política de promoción de empleo del ejecutivo andaluz y que se integran dentro del presupuesto de los programas de fomento del empleo.

\subsection{Medidas orientadas a la Demanda de trabajo}

En este apartado se van a desarrollar, siguiendo el esquema propuesto en el cuadro $n^{\circ} 1$, los programas de fomento de empleo e inserción profesional y el programa de fomento de la economía social.

a) Programas de fomento de empleo e inserción profesional

El programa presupuestario de fomento de empleo junto con el programa de desarrollo cooperativo y comunitario representan acciones orientadas a incrementar los puestos de trabajo y son el tipo de actuaciones que han predominado durante la década de los ochenta. Posteriormente, cuando se inicia la década de los noventa, este tipo de políticas de fomento de empleo se ven desplazadas por otro tipo de actuaciones dirigidas hacia la oferta, como son las correspondientes al programa de formación profesional ocupacional, tal como se puede observar en el cuadro $n^{\circ} 3$, en el que se muestra la trayectoria presupuestatia de los distintos programas.

El programa de fomento de empleo, en cumplimiento de las intenciones politicas contempladas por el ejecutivo andaluz en los distintos planes de desarrollo, ha potenciado unas actuaciones específicas tendentes a facilitar la inserción laboral de 
los colectivos desfavorecidos, a fomentar la creación de empleo estable, y a potenciar las estructuras de apoyo al empleo que favorezcan el desarrollo local ${ }^{6}$. Más concretamente, la intervención del gobierno andaluz se ha centrado para su ejecución en distintos grupos de actuaciones: a) inserción socio-laboral de los jóvenes, b) apoyo a la contratación de trabajadores mayores de 25 años que se encuentren en situación de desempleo prolongado, c) ayudas a proyectos que generen o mantengan empleo, d) apoyo al empleo de la mujer, e) apoyo a la formación en prácticas para los jóvenes de la ciudad, f) actuaciones específicas en colaboración con las Corporaciones Locales, y g) apoyo a las iniciativas locales de empleo.

En este marco se le otorga una especial importancia al colectivo de jóvenes y desempleados de larga duración. De hecho, dos importantes impulsos presupuestarios del total de actuaciones de fomento de empleo, como son los de los años 1987 y 1988, se deben a la inclusión de medidas relacionadas con los sub-programas de "Andalucía Joven" y "Parados de larga duración".

Junto con este tipo de actuaciones de fomento de empleo, pero al objeto de potenciar las'referidas a la lucha contra las desigualdades sociales, acciones que se venían produciendo dentro del programa de fomento de empleo, el ejecutivo andaluz desarrolló, coincidiendo con la tercera legislatura, una serie de medidas específicas de inserción profesional de colectivos concretos con el fin de complementar las medidas de fomento de empleo genéricas que venían desarrollándose.

Estas medidas surgen, en parte, como consecuencia de la firma de un primer acuerdo en 1990 sobre el Diálogo Social entre la Junta de Andalucía y las centrales sindicales mayoritarias. Con este acuerdo, las partes se comprometían a llevar a cabo una serie de actuaciones y, en concreto, se recogía el compromiso sobre la necesidad de crear el Programa de Solidaridad ${ }^{7}$. Con ello se pretendia la integración de las capas marginadas a través de acciones tendentes a la educación permanente de adultos, formación profesional ocupacional y reciclaje para los mayores de 25 años, formación profesional ocupacional y educación permanente para jóvenes comprendidos entre los 16 y 25 años, y participación en empleo temporal fomentado por las diferentes administraciones públicas para trabajos de interés social. Además, se contemplaba la posibilidad de un ingreso mínimo de solidaridad como última medida que se dirigiera a contribuir a la cobertura de las necesidades más esenciales de estas personas.

6. A lo largo de los años se han ido desarrollando normas tendentes a la regulación de los distintos programas de fomento de empleo, entre los que cabe destacar el Decreto 220/84, Decreto 142/1985 y el Decreto 61/1991.

7. A tenor de este acuerdo se procedió a regular, a través del Decreto 400/90 de 27 de noviembre, el Programa de Solidaridad de los andaluces para la erradicación de la marginación y desigualdad en Andalucía. 
Cuadro $n^{\circ} 3$

PROGRAMAS DE PROMOCIÓN DE EMPLEO(*).
Datos sobre obligaciones reconocidas

\begin{tabular}{|c|c|c|c|c|c|c|c|c|c|}
\hline \multicolumn{2}{|c|}{$\begin{array}{c}\text { Fomento } \\
\text { Empleo (1) }\end{array}$} & \multirow{2}{*}{$\begin{array}{c}\text { Inserción } \\
\text { Prof. (2) } \\
\text { Miles ptas. }\end{array}$} & \multicolumn{3}{|c|}{$\begin{array}{c}\text { Fomento } \\
\text { Cooperativismo }\end{array}$} & \multicolumn{2}{|c|}{$\begin{array}{l}\text { Form.Prof. } \\
\text { Ocupacional }\end{array}$} & \multicolumn{2}{|c|}{ Total } \\
\hline Miles ptas. & Variac. & & Variac. & Miles ptas. & Variac. & Miles $\mathrm{pt}$ & & Variac. & Variac. \\
\hline 1985 & 779.725 & - & - & - & 85.359 & & - & - & \\
\hline 1986 & 2.967 .311 & 280,6 & - & - & 866.581 & 915,2 & - & - & 62,9 \\
\hline 1987 & 4.573 .271 & 54,1 & - & - & 826.780 & $-4,6$ & - & - & 111,6 \\
\hline 1988 & 9.479 .524 & 107,3 & - & - & 582.857 & $-29,5$ & - & - & 111,4 \\
\hline 1989 & 11.449 .597 & 20,8 & - & - & 643.682 & 10,4 & - & - & 29,3 \\
\hline 1990 & 16.659 .847 & 45,5 & - & - & 1.182 .383 & 83,7 & - & - & 31,5 \\
\hline 1991 & 9.540 .172 & $-42,7$ & 5.783 .134 & - & 1.457 .105 & 23,2 & 4.017 .204 & - & 41,3 \\
\hline 1992 & 8.799 .704 & $-7,8$ & 4.512 .518 & $-22,0$ & 2.265 .173 & 55,5 & 3.219 .292 & $-19,9$ & 11,7 \\
\hline 1993 & 12.051 .888 & 37,0 & 6.123 .738 & 35,7 & 2.397 .531 & 5,8 & 6.758 .991 & 110,0 & 0,0 \\
\hline 1994 & 16.056 .222 & 33,2 & 6.323 .393 & 3,3 & 2.324.864 & $-3,0$ & 11.374 .366 & $68 ; 3$ & 41,9 \\
\hline 1995 & 10.072 .223 & $-37,3$ & 3.957 .521 & $-37,4$ & 1.353 .100 & $-41,8$ & 21.735 .300 & 91,1 & $-9,1$ \\
\hline 1996 & 11.661 .000 & 15,8 & 4.346 .200 & 9,8 & 2.553 .400 & 88,7 & 24.779 .300 & 14,0 & 16,3 \\
\hline 1997 & 14.902 .300 & 27,8 & 2.239 .900 & $-48,5$ & 4.375 .200 & 71,3 & 27.016 .900 & 9,0 & 11,0 \\
\hline
\end{tabular}

Fuente: Presupuestos de la Comunidad Autónoma, Cuentas Generales de la C.A. y elaboración propia.

( ) Hasta 1991 tan sólo se acometieron de forma expresa los dos primeros (Programa de Fomento del Empleo y Programa de Fomento de la Economía Social), puesto que dentro de programa fomento de empleo se incluyeron acciones de formación profesional ocupacional e inserción profesional que más tarde constituirian programas indepiendientes.

(Los datos referidos a los años 1996, 1997 corresponden a créditos iniciales) ${ }^{\mathrm{a}}$.

Dichas acciones responden a la necesidad de compensar los trastornos sociales que genera el desempleo. Es evidente que la continuidad de los empleos ásí creados no se vincula con las necesidades de provisión de bienes y servicios públicós; sino con su capacidad para compensar parcialmente el deterioro social. El incremento presupuestario de estas medidas a principio de la década de los noventa, coincidiendo con la caída del ciclo económico y su especial repercusión en Andalucía, delata su naturaleza de medidas de urgencia social. Así, tal como puedè apreciarse en el cuadro $n^{\circ} 3$, en el que se muestra la trayectoria presupuestaria de

8. Para ello, se han tomado los datos sobre obligaciones reconocidas, es decir; lo, realménté comprometido, debido a las modificaciones que los presupuestos sufren en sus distintás fáses del gastơ. No obstante, a fin de proyectar estos datos en un periodo más prolongado en el tiempo, para lośtânos 1996 y 1997, se han considerado los datos sobre créditos iniciales. 
cada uno de los programas de promoción de empleo, el programa de fomento de empleo y el de inserción profesional son programas que se ven afectados positivamente en los años de crisis, tal como se ve reflejado con el incremento que se observa en 1993 y 1994, años en que Andalucía sufre con especial intensidad la crisis económica de principios de los noventa.

En general, este tipo de medidas de fomento de empleo e inserción profesional han supuesto bien la prestación de un salario mínimo por la contraprestación de un trabajo cuya duración ha oscilado entre los seis y doce meses, o bien una renta mínima de inserción social, que no han supuesto motivación ni movilización de individuos $y$, en muchos casos, no ha producido una inserción laboral o formativa. En este sentido, este tipo de situación ha podido tener un efecto distorsionador en la oferta de trabajo andaluza (en lo que se refiere a movilidad, rotación laboral, etc.) y ha podido contribuir a que los trabajadores tiendan a enquistarse en una situación de precariedad.

b) Programa de fomento de la economía social

Entre el conjunto de medidas selectivas que incentivan la ocupación de determinados colectivos o, de forma general, el empleo, se encuentran también otras acciones vinculadas a fomentar la iniciativa empresarial con el objeto de crear empleo. En este sentido, las empresas de economía social son un buen instrumento para cumplir con dicho objetivo, no sólo en cuanto a que crean empleo, sino también, porque en periodos de crisis económica son un favorable mecanismo para el mantenimiento de puestos de trabajo (Barrera, 1994).

No será hasta 1985, una vez promulgada la Ley 2/1985 de 2 de mayo, cuando se establezcan los primeros programas autonómicos andaluces de promoción y estímulo al cooperativismo9. Aunque previamente, en 1984, con la Orden del 15 de noviembre del 1984, se establecieron ayudas para el fomento del empleo cuyos beneficiarios eran los socios de las Sociedades Anónimas Laborales y las Sociedades Cooperativas ${ }^{10}$. Dichos programas de fomento de la economía social han estado integrados por una serie de actuaciones consistentes en subvenciones

9. El gobierno autonómico en virtud del art. 13.20 del Estatuto de Autonomía, y de acuerdo con lo esstablecido en la Constitución en su art. 129.2, establece que los poderes públicos fomentarán, mediantè legislación adecuada, las sociedades cooperativas.

10. Esto era consecuencia de las medidas proyectadas en los presupuestos relativos al fomento de la economía social en los que se diseñaban: por un lado, la concesión de subvenciones, por ejemplo. a actividades relacionadas por la promoción, formación y asistencia técnica a cooperativas, a trabajadores por cuenta ajena que deseen constituirse como trabajadores autónomos y; por otro lado, la elaboración de planes y programas para sectores específicos, como por ejemplo el textil. 
económicas ligadas a la inversión, ayudas para la asistencia técnica y gerencial a las Cooperativas, ayudas para la formación y divulgación cooperativa y ayudas para. el fomento del asociacionismo cooperativo.

Este tipo de actuaciones se ha visto respaldado por un presupuesto que ha porderado de muy diferente forma dentro del programa de política de fomento de empleo llevada a cabo por la Junta de Andalucía. Así, de representar el $20 \%$ del total de los programas de fomento de empleo en los primeros años coincidiendo con el primer plan de desarrollo para el periodo 1984-86, pasó a ocupar una posición más marginal durante el desarrollo del segundo plan de desarrollo (1987-90), para después invertirse la tendencia con la implantación del tercer plan (1991-94) (ver cuadro $n^{\circ} 3$ ).

Sin lugar a dudas, dichas acciones han tratado de responder a la necesidad de crear empleo y compensar los trastornos sociales que genera el desempleo en sectores poblacionales marginales, dada la filosofía de la que se impregnan este tipo de empresas. Por principio, las empresas de economía social son entidades locales de amplia base participativa, que actúan como un instrumento prioritario en la lucha contra el desempleo y la exclusión social permitiendo la integración social y laboral de colectivos de difícil inserción o en riesgo de exclusión social, como jóvenes, mujeres, parados de larga duración o los mayores de 45 años.

2.2. Medidas orientadas a la oferta de trabajo: Programa de formación ProfesioNAL OCUPACIONAL

Cuando se diseña una política de empleo centrada en la oferta de formación a los desempleados se está asumiendo implícitamente una serie de argumentos sobre la variable determinante del empleo y sobre la propia naturaleza del paro. En concreto, el objetivo que envuelve el programa de formación profesional ocupacional se centra en mejorar la adaptación del capital humano andaluz a las características de la demanda de trabajo, asegurando una formación de calidad que facilite el acceso al empleo atendiendo a las demandas de formación de los distintos sectores y actualizando los conocimientos profesionales de los trabajadores.

Por lo que respecta a las acciones del gobierno andaluz, hay que esperar hasta finales de la década de los ochenta para describir un cambio de tendencia en la instrumentación de la política de empleo. El programa de formación profesional representa un tipo de actuación que venía desarrollándose con anteriotidad a 1990 dentro del programa de fomento de empleo aunque con un carácter más residual. Sin embargo, coincidiendo con la remodelación que se produce en 1990 como consecuencia de la tercera legislatura y a tenor de lo manifestado en el têrcer plan de 
desarrollo económico para Andalucía (PADE 91-94), en el que se asume que el desajuste cualitativo que existe en el mercado de trabajo andaluz es uno de los mayores obstáculos en la solución del desempleo, la Consejería de Trabajo se plantéa en su nueva etapa la gestión y potenciación de la formación ocupacional. Con este fin, se propuso la creación de un nuevo programa presupuestario especialmente destinado a la formación profesional ocupacional, cuyos recursos estarían dirigidos a los jóvenes de 18 a 25 años a través cle cursos de interés profesional.

Además del fomento que recibe la formación profesional en 1990, en 1993 se producirá un nuevo impulso como consecuencia del traspaso de competencias a la Consejería de Trabajo en materia de gestión de la formación ocupacional de las funciones que venía desempeñando la Administración del Estado a través del INEM, en virtud del R.D. 427/1993 de 26 de marzo. Este traspaso supone un incremento notable de las dotaciones presupuestarias dirigidas a la formación profesional ocupacional y que se ven complementadas con las aportaciones del Fondo Social Europeo. De hecho, en el año 1994, este programa se ve afectado por una fuerte inyección presupuestaria que supone prácticamente la duplicación del presupuesto (cuadro $\mathrm{n}^{\circ} 3$ ).

En definitiva, la política de promoción de empleo del ejecutivo andaluz sufre un punto de inflexión importante en 1991. Las actuaciones dirigidas a la demanda se ven desplazadas por otro tipo de actuaciones dirigidas a la oferta, respaldadas por las mayores competencias en materia de formación profesional ocupacional, y por la política de cofinanciación de la Unión Europea para este tipo de actuaciones a través del Fondo Social Europeo. Se puede decir que entrada la década de los noventa, el diseño de la política de empleo andaluza centrada en la oferta de formación a los desempleados parece estar asumiendo una serie de argumentos sobre la variable determinante del empleo y sobre la propia naturaleza del paro, pues suele afirmarse que la causa del desempleo radica en la falta de cualificación, característica definitoria, por otro lado, de la gran parte del colectivo de parados.

Sin lugar a dudas, estas medidas han tenido reflejo en el mercado laboral andaluz. A través del análisis del mismo pretendemos realizar una serie de consideraciones a la política de apoyo directo del ejecutivo andaluz en su lucha contra el desempleo, teniendo en cuenta que son múltiples las variables que confluyen en la evolución del desempleo. El periodo de tiempo considerado para el balance que pretendemos ha sido desde 1981 a 1997, al considerar que hay medidas que tienen su reflejo en el mercado laboral en años siguientes. 
3. Un balance en materia de merCado de trabajo. Algunas consideractones a há INTERVENCIÓN DIRECTA DEL GOBIERNO AUTONOMO

El balance en materia de mercado de trabajo desde que se pusiera en marchai la política de promoción de empleo del gobierno autonómico no ha sido nada halagüeño. No obstante, la evolución del mercado de trabajo nos permite realizaî una serie de consideraciones, unas de carácter general y otras sobre la intervencién: directa del gobierno autonómico:

a) El desempleo sigue siendo un problema profundamente arraigado en la eco: nomía andaluza

A pesar de haber llevado a cabo múltiples intervenciones en el mercado de tra:bajo andaluz, el problema del desempleo sigue constituyendo en Andalucía el obstáculo más importante que entorpece el bienestar socioeconómico de los andaluces.

Sin lugar a dudas, la evolución del mercado de trabajo en la Comunidad: Autónoma andaluza ha estado condicionado por el crecimiento de la población activa $^{11}$, pero esto no justifica que hoy día se tenga una tasa de paro cercana al $30 \%$, y que ésta se sitúe en prácticamente diez puntos por encima de la media. española.



11. La población activa andaluza ha aumentado desde 1980 hasta 1995 en un $35,5 \%$, casi el doble que en España, motivado por el propio dinamismo demográfico de la región y la intensa incorporación de la población femenina al mercado laboral. 
A pesar de que entre 1981 y 1997 el empleo creciera en mayor proporción en Andalucía que en el conjunto de España, la tasa de paro andaluza se aceleró notablemente hasta llegar a rozar el 35\% en 1994. Por consiguiente, ni tan siquiera en là etapa de expansión económica (1987-1991) que vivió Andalucía, con un crecimiento económico con tasas superiores a las producidas en el ámbito nacional, se ve amortiguado el problema del desempleo.

b) Escasa capacidad del sistema productivo de generar empleo

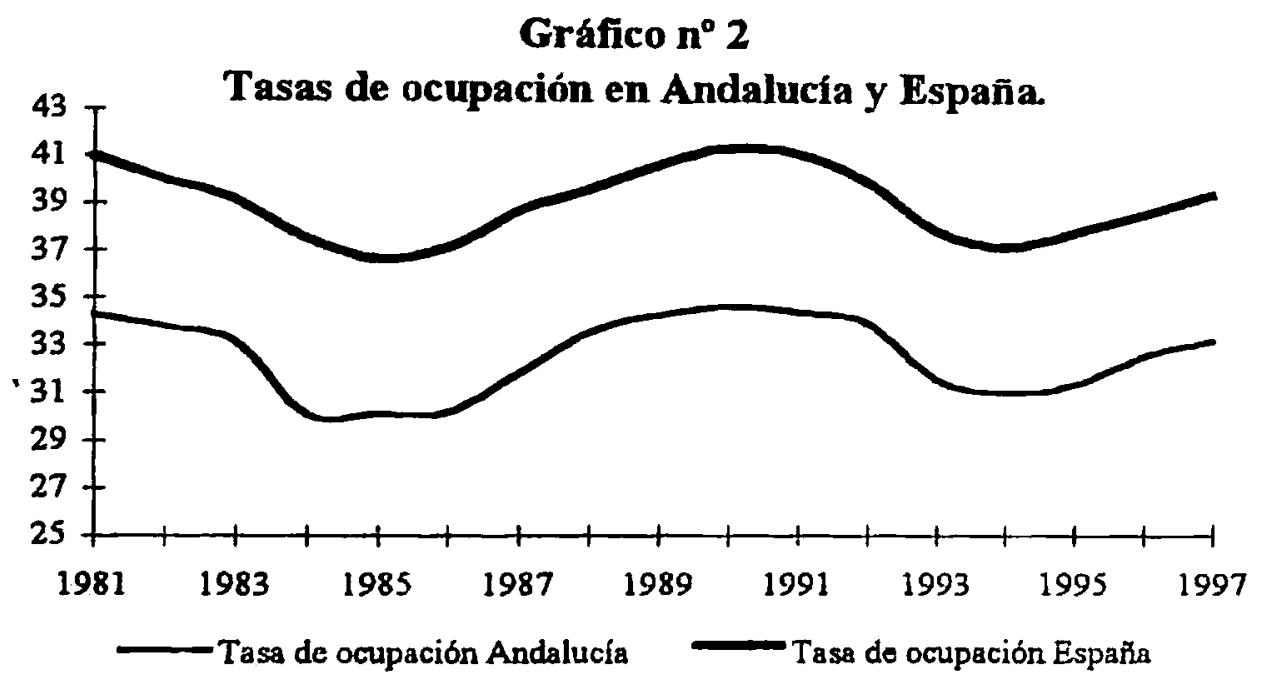

Fuente: BPA, INE. Elaboración propia.

Una de las características de la economía española es su baja elasticidad a la creación de empleo (Segura, 1993). Pues bien, si esto es cierto en el ámbito nacional, parece que lo es más en Andalucía. La tasa de ocupación andaluza no ha logrado aproximarse a la tasa de ocupación española, ni tan siquiera en la etapa de mayor crecimiento de la economía andaluza. Tan sólo ha logrado mantenerse situándose seis puntos por debajo de la tasa española. Coincidiendo con la etapa de mayor crecimiento (1989-1991), la tasa de ocupación llega a registrar sus valores más altos del periodo de estudio ( $34,3 \%$ en 1989 y $34,7 \%$ en 1990), pero al mismo tiempo se alcanzan las mayores diferencias con la tasa de ocupación española (gráfico $n^{\circ} 2$ ).

c) Una ocupación basada en la expansión de las inversiones públicas y empleo público

En general, el empleo por sectores ha cambiado ligeramente en relación con el existente a mediados de los años ochenta. La agricultura ha sido el sector que ha estado expulsando continuamente mano de obra. Por el contrario, el sector servi- 
cios ha estado en continuo aumento conociendo el periodo de mayor expansión entre 1985 y 1990 . Este periodo no sólo fue expansivo para los servicios sino también para la construcción, como consecuencia, en cierta manera, del periodo expansivo en inversiones públicas que se vive en Andalucía a final de los ochenta, por lo que concluidos estos años de fuertes inversiones sufriría un retroceso. Por otro lado, la ocupación del sector industrial sigue el mismo comportamiento que el total de la población ocupada en el ámbito andaluz. Sin bien, en periodos de crisis, la caída en la ocupación industrial se hace más intensa y, por el contrario, en etapas de expansión, la ocupación industrial crece en menor medida (gráfico $\mathrm{n}^{\circ} 3$ ).

Evolución de la poblacín ocupada por sectores.

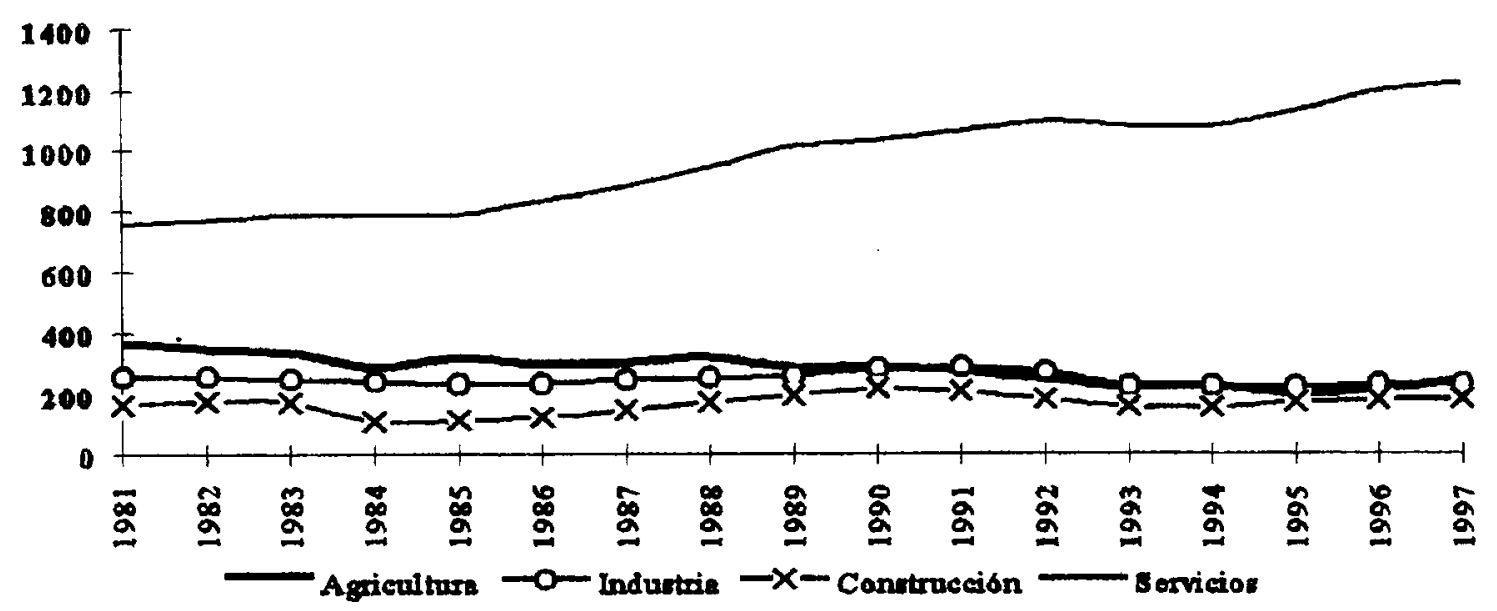

Fuente: EPA, WNE. Eleboración propia.

El resultado final es que para el conjunto del periodo 1981-1997, la ocupación total en la Comunidad Autónoma presenta un aumento del 12,2\%. Este crecimiento está motivado fundamentalmente por el buen comportamiento de los servicios y, en concreto, por el continuo crecimiento del empleo en el sector público. El número de ocupados en el sector público andaluz pasa de 206.689 efectivos en 1983 hasta 340.310 ocupados en 1993, lo que supone un crecimiento de un 64\%. Esto pone de manifiesto que han sido las inversiones públicas y la política pública de empleo las que han logrado incrementar la ocupación en este periodo, aunque, en muchos casos, el aumento de la ocupación se ha materializado en contratos eventuales que de forma coyuntural han intentado paliar el problema del desempleo. 
d) La política de fomento de empleo ha permitido amortiguar las altas tasas de desempleo

El conjunto de medidas integradas dentro del programa de política de fomento de empleo del gobierno autónomo y, principalmente, instrumentadas por el lado de la demanda, han posibilitado una serie de mecanismos para que los grupos de población considerados más desfavorecidos en el mercado laboral tengan una oportunidad de trabajar. Como hemos visto anteriormente y a tenor de las cantidades asignadas a cada una de las medidas, son el grupo de jóvenes y los desempleados de larga duración los más favorecidos.

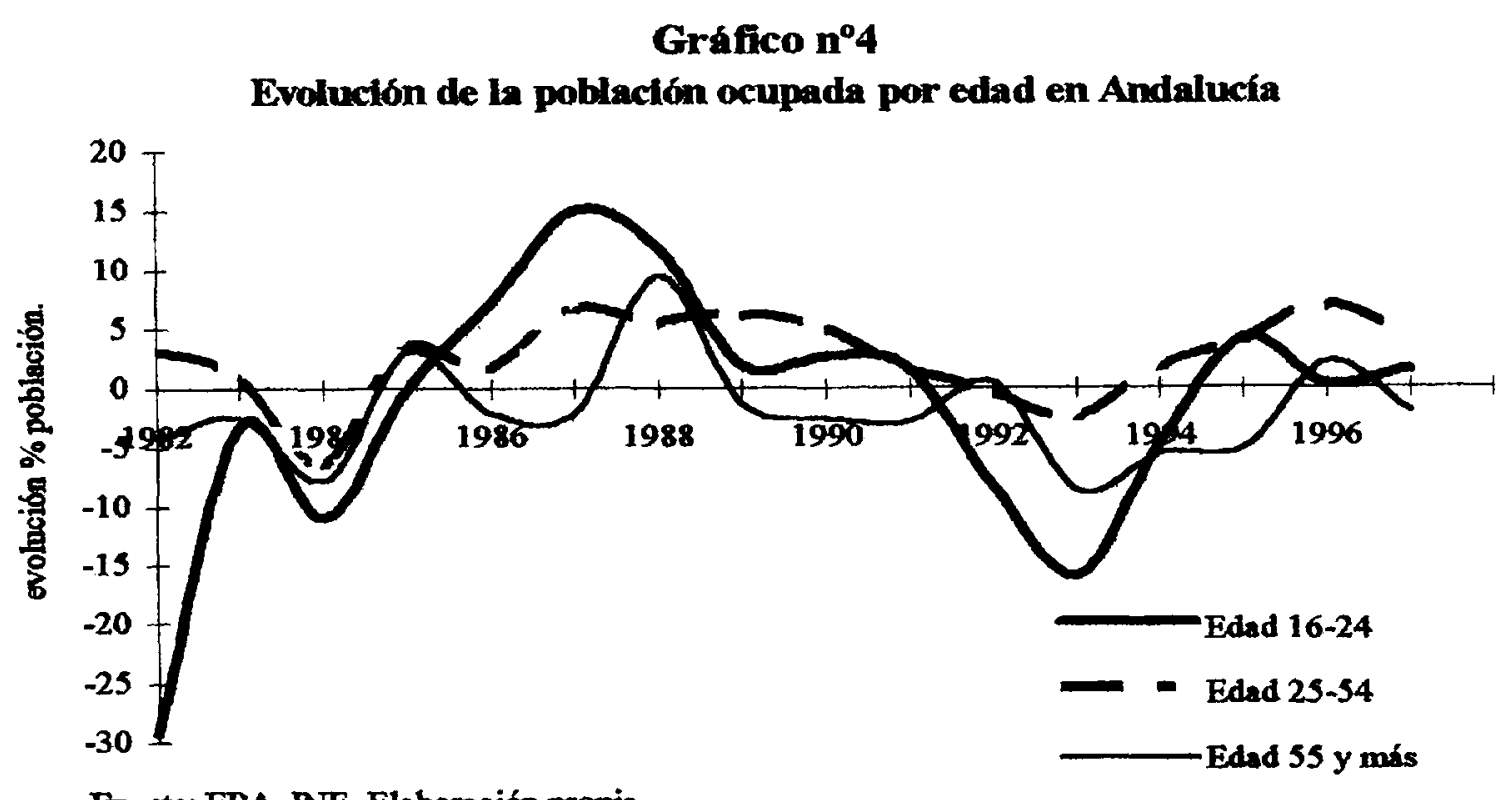

Fuente: EPA, INE. Elaboración propia.

Sin embargo, esta política de fomento de empleo dirigida a favor de la contratación de grupos desfavorecidos no se ha caracterizado por la creación de empleo estable, pues, generalmente, estos empleos han estado vinculados a contratos laborales cuya duración ha estado entre los 6 y 12 meses. En particular, las políticas dirigidas al sector juvenil sólo han solucionado el problema de forma eventual. De hecho, en un momento dado, esto pudo contribuir a amortiguar las cifras de paro en este segmento de la población, puesto que desde 1984 la ocupación de este sector poblacional evoluciona de forma positiva (entre 1985 y 1990 el número de ocupados con edades comprendidas entre 16 y 24 crece un 43,8\%), coincidiendo en el tiempo con la aplicación de la política de empleo de la Junta de Andalucía diri- 
gida a los jóvenes menores de 25 años (gráfico n ${ }^{\circ}$ 4). Por el contrario, iniciado el año 1990, que coincide con un punto de inflexión en la política de la Junta de Andalucía en materia de empleo (la política de empleo se centra principalmente en la formación), son los jóvenes entre 16 y 24 años el sector más castigado. De igual forma, en los años 1993 y 1994 se produce un notable incremento en el presupuesto de los programas de fomento de empleo e inserción profesional (que atiende especialmente a los jóvenes), que corresponde con una nueva evolución positiva de la ocupación en este grupo poblacional.

Por tanto, los programas de fomento de empleo e inserción profesional han contribuido a suavizar las altas tasas de paro del sector poblacional juvenil. No obstante, hoy día sigue siendo el sector poblacional más castigado.

e) El fomento de la economía social y las ayudas al autoempleo: una solución a la creación de empleo estable

Las Cooperativas de Trabajo Asociado y las Sociedades Anónimas Laborales se han convertido en los protagonistas del periodo considerado como salida al problema del desempleo.

En el caso de la región andaluza, el núcleo fundamental de la economía social lo constituyen las cooperativas, con especial protagonismo las de trabajo asociado. Del total de 3.503 cooperativas censadas, 1.768 corresponde a trabajo asociado, representando éstas un volumen total de empleo de 19.155 ocupados en 1996 (Dirección General de Cooperativas, 1996).

Cuadro $n^{\circ} 4$

Evolución de las sociedades anónimas laborales 90-95

\begin{tabular}{lcccccc}
\hline & 1990 & 1991 & 1992 & 1993 & 1994 & 1995 \\
\hline$N^{\circ}$ sociedades creadas & 17 & 47 & 73 & 107 & 125 & 97 \\
$N^{\circ}$ socios & 85 & 431 & 482 & 855 & 914 & 1139 \\
\hline
\end{tabular}

Fuente: Anuario Estadístico de Andalucia. Consejería de Trabajo. Elaboración propia. 




Otra parte integrante de la economía social son las Sociedades Anónimas Laborales. De las 3.906 empresas de economía social en Andalucía, 403 corresponden a las Sociedades Anónimas Laborales. Tal y como se puede apreciar en el cuadro $n^{\circ} 4$, donde se presenta la evolución de las mismas durante 1990-1995, éstas han tenido un incremento sucesivo en el periodo analizado.

Esto nos hace presumir que el fomento de la economía social se ha convertido en un instrumento idóneo de promoción el empleo estable en Andalucía. De hecho, la deplorable situación del empleo andaluz se ha visto aliviada por el relativo buen comportamiento de los empleos no asalariados (empleadores, donde estarian incluidos los miembros de las cooperativas) (gráfico $n^{\circ} 5$ ). Mientras que para España el autoempleo ha decrecido de forma continuada desde 1987 (Sánchez y Morillas, 1993), salvo el paréntesis que supuso 1992. En cambio, en Andalucía, el autoempleo creció entre 1986 y 1990, coincidiendo en el tiempo con el impulso que las autoridades autonómicas le dan al fomento de la economía social, experimentando ligeras fluctuaciones en los años siguientes.

\section{Consideraciones finales}

Desde hace varias décadas, en Andalucía se viene produciendo un desajuste entre la oferta de trabajo y la capacidad de generación de empleo del aparato productivo regional. Con el paso de los años transcurridos de gobierno autonómico andaluz tan sólo se ha logrado mejorar eventualmente la situación, pero ni siquiera en los momentos de mayor crecimiento económico la tasa de paro en Andalucía 
ha podido descender significativamente, lo que pone de manifiesto las raíces pro: fundas del problema y se sigue evidenciando como el principal desafío de la eco:nomía andaluza.

Las actuaciones de intervención directa del ejecutivo andaluz en materia de promoción de empleo tan sólo han logrado amortiguar las altas tasas de desempleo: de la economía andaluza. Las medidas integradas dentro de las actuaciones de fomento de empleo e inserción profesional han permitido crear puestos de traba: jo vinculados a una subvención que se recibe por un espacio de tiempo determinado, pero no a la necesidad de mano de obra del empleador. Aún así, a pesar de no crearse empleo estable, estos instrumentos pueden calificarse de positivos si las: medidas de fomento de empleo en el sector público se vinculan con las necesidades de provisión de bienes y servicios públicos, y si permiten el acceso a unos, puestos de trabajo a colectivos con serios problemas de empleo e integración social, cumpliendo una evidente función social. Por tanto, las actuaciones acometidas en este área no son fácilmente evaluables. Si bien, es cierto que en ausencia de todas estas prestaciones en favor del empleo, el número de parados sería mayor. Por ello, si las políticas asumidas no resuelven el problema del desempleo, al menos, contribuyen a atenuarlo.

En el caso específico de Andalucía, este tipo de política de carácter asistencialista del gobierno autonómico junto con las transferencias procedentes del gobierno central han logrado que la renta familiar disponible y la renta familiar disponible per cápita de los andaluces mejorara durante estos años (Torres, 1995). No obstante, esto ha podido contribuir, en cierta manera, a que los patrones de la desigualdad sean más palpables (Ibid., 1995), a pesar de estas mayores inyecciones de gastos sociales a través de los programas de fomento de empleo para paliar la situación de los colectivos sociales más desfavorecidos. Ante esto, la pregunta que hay que plantearse es en qué medida dichas transferencias sirven para crear o desarrollar auténticas fuentes de riqueza que supongan la transformación de las zonas receptoras. En este sentido, Cuadrado (1990) sostiene que los procesos redistributivos de carácter asistencial no sólo pueden redundar en un menor dinamismo y capacidad de innovación por parte de las regiones receptoras, sino que, a lo largo plazo, suelen ser la mejor garantía de su permanencia en el atraso económico. Así, la recuperación de la economía es de pobre resultado si no se acaba con la manifiesta incapacidad de la Comunidad Autónoma andaluza para crear empleo y corregir los patrones de desigualdad. 
Otro bloque importante de medidas han sido las de fomento de la economía social. En los últimos tiempos se ha producido un aumento de este tipo de empresas, que ha venido en parte promovida por una legislación favorecedora o promotora de la misma. Este tipo de actuaciones ha permitido que las empresas de economía social avancen y se consoliden como un sector especialmente importante dentro del panorama económico andaluza y, al mismo tiempo, como un instrumento generador de empleo.

Respecto a la política de intervención directa del ejecutivo andaluz dirigida a mejorar la adaptación del capital humano andaluz a las características de la demanda de trabajo, asegurando una formación de calidad que facilite el acceso al empleo, se concreta en un conjunto de medidas que resultan positivas a pesar de que los resultados se obtienen más a largo plazo. Sin embargo, no se puede aspirar, con la política de formación, a modificar sustancialmente, en el plazo más corto posible, una situación de desempleo masivo. Aún cuando las medidas de fomento de empleo por la vía de la oferta, para conseguir un resultado positivo, pasan necesariamente pòr modernizar y diversificar el tejido productivo. Una política formativa junto con un tejido productivo estancado, provocaría una población activa desempleada altamente cualificada.

\section{REFERENCIAS BiBLIOGRÁFICAS}

CASTILLO S., DUCE, R.M. y JIMENO, J.F. (1994): "El mercado de trabajo en Andalucía: los factores determinantes del paro masivo y persistente". Boletín Económico de Andalucía, $n^{\circ}$ 18. Consejería de Economía y Hacienda.

CONSEJERÍA DE TRABAJO Y BIENESTAR SOCIAL (varios años): Memoria de actividades de la Consejería de Trabajo y Bienestar Social. Junta de Andalucía.

CUAADRADO, J.R. (1990): "Una nota en torno a la evolución de las disparidades regionales en España". Economistas, no 45-46. Madrid.

DELGADO, M y ROMÁN, C. (eds.), (1995): Ocho Análisis de la Economía Andaluza. Instituto de Desarrollo Regional. Sevilla.

DIRECCIÓN GENERAL DE COOPERATIVAS (1996): Censo de Sociedades Cooperativas Andaluzas y Sociedades Anónimas Laborales en Andalucía. Junta de Andalucía, Sevilla.

BARRERA, J.J. (1994): "El papel de la Administración Central en el Fomento de la Economía social". Boletin ICE, No 729.

GONZÁlEZ, F. y VILLALBA F. (coords.) (1997): Retos de futuro para Andalucía empleo y paro. Universidad de Málaga, Debates.

INSTITUTO NACIONAL DE ESTADÍSTICA (varios años): Encuesta de población activa. Madrid. 
JUNTA DE ANDALUCía (varios años): Presupuestos de la Comunidad Autónoma de Andalucía. Sevilla.

JUNTA DE ANDALUCÍA (varios años): Cuentas Generales de la Comunidad Autónoma dé. Andalucía. Sevilla.

JUNTA DE ANDALUCÍA (varios años): Memorias de Actividades de la Consejería de Trabajo, Sevilla.

MORILLAS, A. y SÁNCHEZ, J. (1990): "Situación actual y perspectivas del mercado de trabajo en Andalucía". Revista de Estudios Regionales, $\mathrm{n}^{\circ} 28$.

OCDE (1996): Perspectivas del empleo. Ministerio de Trabajo y Asuntos Sociales. Madrid.

REHN, G. (1989): Ensayos sobre la política de empleo activa.Centro de publicaciones del Ministerio de Trabajo y Seguridad Social. Madrid.

SEGURA, J. (1993): "Algunas reflexiones sobre la reforma del mercado de trabajo". Cuadernos de Información Económica, $n^{\circ} 75$.

SÁNCHEZ y MORILLAS. A. (1993): "Actividad , empleo y paro en España según la encuesta: de población activa", Revista de Estudios Regionales, $\mathrm{n}^{\circ} 36$.

TOHARIA, L. (dir.) (1998): El mercado de trabajo en España. Ed. Mc Graw Hill.

TORRES, J. (1995): "Crecimiento económico y distribución de la renta en Andalucía. Viejos: problemas, excusas modernas", en DELGADO, M. y ROMÁN, C. (eds.). Ocho Análisis de La Economia Andaluza. Instituto de Desarrollo Regional, Sevilla. 